Solid State Communications, Vol. 83, No. 11, pp. 899-903, 1992.

\title{
LANDAU-CUTKOSKY RULES, SCALE INVARIANCE, AND THE MARGINAL FERMI LIQUID
}

\author{
J.W. Rasul and R. Hoogerbeets
}

Department of Physics, The University of Michigan, Ann Arbor, MI 48109-1120, U.S.A.

(Received 27 December 1991; in revised form 4 May 1992 by A.A. Maradudin)

\begin{abstract}
With the aid of the reduced graph expansion relating different order multiple interaction vertices and the exact spectral densities, we show that a strongly coupled Fermi system can allow solutions for the spectral density which scale under simultaneous scale changes in the energy and temperature. This implies a functional form for the spectral density which falls off as a power $\mu$ of frequency between 0 and -1 . The theory describes $Z_{k_{r}}=0$ quasiparticles with a Fermi surface consistent with Luttinger's theorem while the spin and charge susceptibilities have imaginary parts of the form $(\omega / T) T^{1+2 \mu}$ at low energies. The approach reproduces some features of both the Luttinger liquid and marginal Fermi liquid phenomenologies.
\end{abstract}

ONE OF THE MOST important issues facing condensed matter theory today concerns the nature of the normal state of high temperature superconductors. The peculiar properties of this normal state suggest that there exist solutions to the interacting electron problem that are drastically different from the Fermi liquid description. However, there exists at present no general framework analogous to microscopic Fermi liquid theory within which such systems can be placed. In this paper we address this issue by examining the overall structure of pertur-bation theory for an interacting one band model at general order. We shall prove rigorously the existence of scale invariant solutions for the spectral density which dominate the single particle spectrum in the limit of strong coupling and are valid solutions regardless of the physical dimension.

Our approach includes the two dominant schools of thought which bypass the conventional Fermi liquid description. One of these, the Luttinger liquid approach [1], proceeds by analogy with the one dimensional Hubbard-Heisenberg model, which through bosonization, the charge and spin fluctuations exhaust the spectrum near the Fermi surface. In the other approach, the "marginal Fermi liquid" picture, the width of the excitation spectrum is proportional to the temperature, or the frequency, whichever is larger. Moreover, the extremely broad nature of the quasiparticle damping is thought to arise from singularities in the three particle scattering amplitude [3].

In this paper we use the analytic properties of perturbation theory to discuss the properties of all intermediate states, with multiple electron-hole pairs, on an equal footing. Our approach derives ultimately from that developed in high energy theory in the 1960s (the S-matrix approach) for treating the strong interactions [4]. In this pre-QCD era theorists used such general properties as unitarity and analyticity of the S-matrix to derive relations between the real and imaginary parts of scattering amplitudes and crosssections.

The underlying basis for such theoretical approaches was provided by the Landau-Cutkosky rules [5] whereby the imaginary part of a scattering amplitude was related, by making cuts across intermediate many-particle states, to products of higher order vertices [6]. Langer formulated the interacting electron problem in such terms [6] and the technique has been used to discuss impurity scattering [6] and the $T^{3} \ln T$ component of the specific heat of a Fermi liquid [7], amongst other problems.

Our treatment of a general interacting one band model will follow that of Larsen [8] and Mattuck [9] who used the reduced graph expansion to show the presence of strong coupling scale-invariant solutions to the single impurity Kondo problem [10]. Being a two-fermion problem the Kondo problem possesses two different spectral densities and Mattuck's scale invariant ansatz assumed that the conduction electron spectrum scaled in a trivial fashion (connected with the fact that the conduction states are essentially unaffected by the local exchange 
interaction). For a lattice two band model this can no longer be assumed and we therefore proceed with the one band case since the diagrammatic formu-lation is simpler and statements concerning scale invariance can be made more precise.

We shall show that scale invariance in the strong coupling regime leads to a power law dependence of the spectral density on energy, on condition that the frequency and temperature scale together. The reduced graph expansion then links up the power law variation of the spectral density with higher order vertices, including the irreducible self energy. This in turn places bounds on the power law falloff of the spectral density which are consistent both with angleresolved photoemission experiments (ARPES) [11] and exact results for the Hubbard-Heisenberg model in one dimension [1].

Our starting point is the reduced graph expansion rules as given by Langer [6] or in the appendix of Mattuck [9] whereby the imaginary part of a scattering amplitude involving $n$ fermion lines can be written in terms of higher and lower order endpiece vertices for a given number of intermediate particle or hole states. Each of these intermediate states involves merely the spectral function $A(x, T)$ multiplied by the Fermi function for particles or holes (in the following $\{x\}$ denotes the set of internal frequencies, and the set of external frequencies is denoted by $\{y\}$ ), integrated over the internal frequency. The endpieces which themselves are made up of lower order Feynman diagrams are denoted by ${ }^{R, A} \Gamma_{n, m}$ where $n$ denotes the number of lines entering and $m$ denotes the number of lines leaving, and $R(A)$ denote retarded (advanced) vertices.

The spectral densities and vertex endpieces are themselves momentum dependent - however since this is not germane to our analysis we do not make these dependencies explicit. Finally, energy conservation is ensured with the aid of a delta function, and an overall inverse Fermi or Bose function whose argument as the sum of all external energies is also included. The resulting expression for the imaginary part of an $n$th order endpiece shown in Fig. 1 is

$$
\begin{gathered}
\operatorname{Im} \Gamma_{n}^{i^{\prime}}\left\{y_{2 n}\right\}=\sum_{i j} A_{i j} \sum_{\delta, p}\left[\exp \left(\frac{\sum y}{T}\right)+(-1)^{p-1}\right](-1)^{h} \\
\int \prod_{r=1}^{p} \mathrm{~d} x_{r} A\left(x_{r}, T\right) f\left[\left( \pm x_{r}\right)\right] \delta\left(x_{1}+x_{2}+x_{p}-\sum y\right)^{R} \\
\quad \times \Gamma_{n-\delta, p}\left\{y-n_{\delta}, x_{p}\right\}^{A} \Gamma_{p, n+\delta}\left\{x_{p}, y_{n+\delta}\right\}
\end{gathered}
$$

where the factor $A_{i, j}$ refers to the sign of a particular combination of endpieces (themselves labeled by $i$ and $j$ ). The symbol $p$ is the number of intermediate

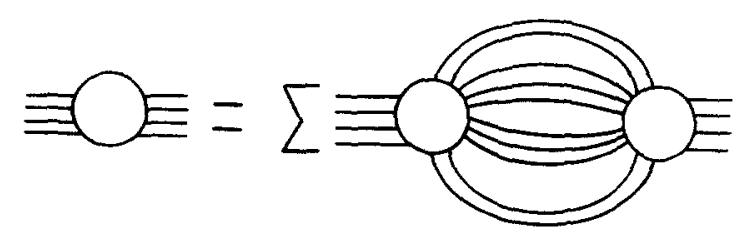

Fig. 1. The diagrammatic relation between a general vertex with $n$ fermion lines entering and leaving, and other endpiece vertices with $n-\delta$ and $n+\delta$ lines entering and leaving the graph. Of the $p$ internal lines, $h$ of these are hole lines.

states and $h$ is the number of hole propagators. It is particularly important to note the role played by the Fermi functions since these dictate that, under scaling, both energy and temperature have to be scaled together. Another point to notice is that the spectral functions in the reduced graph expansions are the exact ones, and the expression (1) holds regardless of the model (Hubbard, extended Hubbard) invoked.

The crucial aspect of equation (1) is that it is invariant under a simultaneous rescaling of all the energy variables and the temperature by a scaling factor $b$ as long as the endpieces and spectral density scale are as follows

$\Gamma_{n}\left\{y_{n}\right\}=b^{\alpha+\beta n} \Gamma_{n}\left\{y_{n} / b\right\}$

$A(x, T)=b^{u} A(x / b, T / b)$,

and the components are determined from the balance of the right and left hand sides. This in turn requires the coefficient of $p$ in the exponent to be zero. Consequently the $n$th order vertex scales as follows:

$\Gamma_{n}\left\{y_{n}\right\}=b^{1-n(1+\mu)} \Gamma_{n}\left\{y_{n} / b\right\}$,

which leaves only the parameter $\mu$ undetermined. Note that such scaling of the endpiece vertices implies that all Feynman diagrams with a specified number of lines entering and leaving scale in the same manner. The scaling (3) requires that $A(x, T)$ take the form

$A(x, T)=\tilde{f}(x / T) T^{\mu}$

and $\tilde{f}(0)$ is non zero, and homogeneity in $x$ and $T$ requires that for large arguments $\tilde{f}(y) \simeq y^{u}$. The exponent $\mu$ can be bounded if we realize that the power law decay of the self energy is simply related to the lowest order irreducible vertex $\Gamma_{1}(y, T)$. This is found from equation (2) to be $\Gamma_{1}(y) \sim y^{-\mu}$ in the limit of zero temperature. To be consistent with the above expression for the spectral density it is necessary that the self energy wins out over the linear term and the band energy $\epsilon_{k}$ in the expression for the single particle Greens function $G(k, \omega)=\left[\omega-\epsilon_{k}-\Sigma(\omega)\right]^{-1}$ in the limit of low 


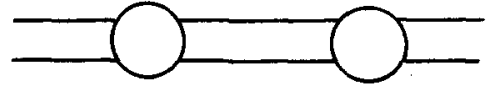

Fig. 2. Reduced graph for the two particle vertex involving only two particle intermediate states.

energy (relative to a cutoff energy scale to be determined below). This then requires that $\mu$ lies between zero and minus one. Of course at low enough frequencies the band term will become important and scale invariance breaks down but we shall show that this regime becomes arbitrarily small relative to the scaling region as the strong coupling regime is approached.

Normalization of the spectral function is then only possible if a high energy cutoff $\Delta$ is imposed on the spectrum, beyond which the spectral function is taken to be zero. As a result the spectral function and self energy take the form

$A(\omega, 0)=\frac{2}{(1+\mu)^{-1}} \frac{1}{\Delta}(\omega / \Delta)^{\mu}$,

$\sum(\omega, 0)=\Delta(\alpha+i \beta)(\omega / \Delta)^{\mu}$

where $\alpha$ and $\beta$ are constants and the KramersKronig relation has been used to show that the real and imaginary parts of the self energy have the same energy dependence. As a result the condition that $\Sigma$ be larger than $\omega-\epsilon_{k}$ in the single particle Greens function requires that $\Delta \gg \epsilon_{k}$. This scale can only be determined in principle by solving completely but we can estimate $\Delta$ for a particular model by truncating equation (1) at the two particle level and seeing over what energy range the scaling form for the two particle vertex remains valid. As equation (1) describes the strong coupling limit correctly all the intermediate state contributions are equally important and should all contain the same energy dependence. Hence the two particle state at least represents qualitatively the energy dependence of the full solution to equation (1).

We therefore turn to the one band Hubbard model and examine the two particle graphs shown in Fig. 2 for which the Landau-Cutkosky rules give

$$
\begin{gathered}
\operatorname{Im} \Gamma_{2}(\omega, T)=\left(\mathrm{e}^{\beta \omega}-1\right)\left|\Gamma_{2}(\omega, T)\right|^{2} \int \mathrm{d} 1 A(1) f(1) \\
\int \mathrm{d} 2 A(2) f(-2) \delta(\omega+1-2) .
\end{gathered}
$$

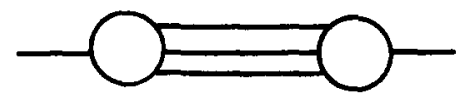

Fig. 3. Self-energy contribution from the two particle vertices.

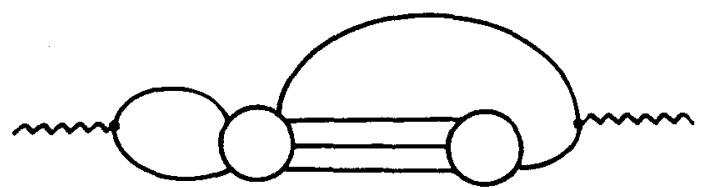

Fig. 4. Graphs contributing to the dynamic spin susceptibility. Wiggly lines denote the external field.

At large energies the bare interaction $U$ has to be recovered and the usual parquet type solution ensues

$\Gamma_{2}(\omega)=U /[1-U \chi(\omega)]$

where $\chi(\omega)$ denotes the Lindhard function calculated using the spectral densities given by equation (6). This is readily evaluated and at $T=0$ the low energy form of this expression is

$\chi(\omega) \sim(1 / \Delta)(\omega / \Delta)^{1+2 \mu}$.

Inserting this back into equation (9) we find that for $\omega \gg \Delta$ the scaling form (4) is recovered. However, as the energy is increased the first term in the denominator becomes more important and the scaling behavior breaks down. If this energy is to correspond to the cutoff $\Delta$ then we can see how this determines the limits of the scaling behavior by examining the lowest order contribution to the self energy $\Sigma$ (shown in Fig. 3) which involves three internal lines and the endpieces given by equation (9). For small values of the external energy $\omega$ the scaling behavior given by equations (4) and (7) holds. On increasing $\omega$ to energies of the order of $\Delta(U / \Delta)^{1 /(1+2 \mu)}$, the endpiece $\Gamma_{2}$ approaches a constant and from Fig. 3 this puts an end to the $\omega^{-\mu}$ scaling behavior of $\Sigma(\omega)$. Since this energy is by definition equal to $\Delta$ this implies that $\Delta \sim U$. Hence the interaction strength determines the magnitude of the cutoff and the limit to scaling. From our earlier argument regarding the validity of the scaling approach to be $\Delta \gg \epsilon_{k}$ we see that the scaling behavior in equations (4) and (7) is valid in the strong coupling limit of the Hubbard model.

We now explore further the consequences of the scaling behavior of the spectral density and self energy. For small band energies relative to the temperature a peak is expected at low frequency whose width is proportional to temperature. At band energies much larger than the temperature (as is the case for ARPES measurements) the peak width has more of a quasiparticle character marking the low frequency limit to the scaling behavior, and should track with the band energy $\epsilon_{k}$. However the most important consequences of our analysis concern the frequency dependence in the "incoherent" region at higher frequencies. There we naturally reproduce the 
exponent falloff observed in the ARPES measurements. Furthermore we require that the exponent be between zero and minus one.

Experimentally the long tails appearing in ARPES measurements suggest there is some evidence for a power law behavior with $|\mu|<1$ in $\mathrm{Bi}_{2} \mathrm{Sr}_{2} \mathrm{BaCu}_{2} \mathrm{O}_{8}$ [1, 11, 12] In fact, careful fits of normal and marginal Fermi liquid line shapes (Liu et al. [12]) require a subtraction of an immense inelastic background. Preliminary fits of a power law indicate that $\mu \sim 0.7$ [12].

Theoretical support for the validity of the present approach can be found in one dimension where a combination of exact results and orthogonality catastrophe arguments for the infinite $U$ Hubbard model imply that the spectral function decays with a power law of $-7 / 8[1,11]$. Our results are independent of dimension, and therefore suggest that the Luttinger liquid phenomenology may well be valid in two dimensions. A simple consequence of the self energy (7) is that the Fermi surface (obtained by setting $\omega=E_{k}=0$ in $G(k, \omega)$ ) is the same as in the non-interacting system, while the weight factor $Z_{k}=1-\left.(\partial \Sigma / \partial \omega)\right|_{\omega=E_{k}}$ vanishes at the Fermi surface. The momentum distribution near the Fermi surface varies as a power law $n_{k}-n_{k_{F}} \sim\left|k-k_{F}\right|^{1+\mu}$ again in a manner consistent with Luttinger liquid results $[1,13]$.

A further consequence of the Landau-Cutkosky rules relates to the dynamic spin and charge susceptibilities, which couple external fields to pairs of incoming and outgoing fermion lines. The lowest order insertion to these lines is the graph in Fig. 3, which from equation (8) leads to the appearance of an overall inverse Bose factor, since only a two particle intermediate state is involved. Although higher order insertions involve intermediate states with odd numbers of lines, in order that they should contribute to the susceptibilities the ends of these lines have to be tied together. The result of tying the incoming and outgoing lines to the external field is that overall, cuts can only be made across even numbers of fermion lines (see for example Fig. 4). The result is that all graphs yield an overall inverse Bose factor whose argument is the external frequency. Thus the imaginary parts of the spin and charge susceptibilities have the following linear dependence on frequency at finite temperatures

$\operatorname{Im} \chi_{s p}, \chi_{c h} \sim(\omega / T) T^{1+2 \mu}$,

which is obtained by expanding the inverse Bose factor to leading order in the frequency. The remaining factors in the expressions for $\chi_{c h}$ and $\chi_{s p}$ obtained from the Landau-Cutkosky rules have a finite limit as $\omega \rightarrow 0$. This result is consistent with the large background Raman intensities [14] and shows linearity in frequency on a scale proportional to temperature as in the marginal Fermi liquid hypothesis [2]. The overall prefactor, however, is itself a power of temperature. It would be interesting to test this overall scaling factor by measuring the temperature dependence of the Raman background intensity and linking it with the ARPES data.

In conclusion, we have shown from general considerations, independent of dimensionality about the structure of perturbation theory at general order that the simple observation that the strong coupling regime allows scale invariant solutions leads to a spectral density with a power law falloff as a function of energy. The scaling form of the spectral density extends as far as a cutoff of order of the interaction strength and in the limit of strong coupling should dominate the single particle spectrum. The scale invariant solutions are homogeneous in frequency and temperature and to that extent are consistent with the marginal Fermi liquid hypothesis. Furthermore, the Landau-Cutkosky rules imply that the imaginary parts of the spin and charge susceptibilities are initially linear in frequency on a scale determined by the temperature with a temperature dependent prefactor that determines the variation of the Raman background intensity. Overall, this approach provides a general framework within which the strongly interacting electron problem can be studied. Work is currently in progress on incorporating momentum dependent corrections into the above treatment, with the hope of understanding the effects of dimensionality.

\section{REFERENCES}

1. P.W. Anderson, Phys. Rev. Lett. 65, 2306 (1990).

2. C.M. Varma, P.B. Littlewood, S. Schmitt-Rink, E. Abrahams \& A.E. Ruckenstein, Phys. Rev. Lett. 63, 1996 (1989).

3. C.M. Varms, Private communication.

4. R.J. Eden, P. Landshoff, D.A. Olive \& J.C. Polkinghorne, The Analytic S-Matrix. Cambridge University Press, Cambridge (1966).

5. L.D. Landau, Nuclear Physics 13, 181 (1959); R.E. Cutkosky, J. Math. Phys. 1, 429 (1960).

6. J.S. Langer, Phys. Rev. 123, 997, 1003 (1961).

7. G.M. Carneiro \& C.J. Pethick, Phys. Rev. B11, 1106 (1975).

8. U. Larsen, Proc. 14th Int. Conf. Low Temp. Phys. (Edited by M. Krusius \& M. Vuorio), North-Holland, Amsterdam (1975).

9. R.D. Mattuck, A Guide to Feynman Diagrams the Many-Body Problem, 2nd edition. McGrawHill, New York (1975). 
10. N.E. Bickers, Rev. Mod. Phys. 59, 845 (1987).

11. C.G. Olson, R. Liu, D.W. Lynch, R.S. List, A.J. Arko, B.W. Veal, , Y.C. Chang, P.Z Jiang \& A.P. Paulikas, Phys. Rev. B24, 381 (1990).

12. L.Z. Liu, R.O. Anderson \& J.W. Allen, $J$.
Phys. Chem (to appear), and private communication.

13. M. Ogata \& H. Shiba, Phys. Rev. 41, 2326 (1990).

14. S.L. Cooper \& M.V. Klein, Comments in Condensed Matter Physics 15, 99 (1990). 